\title{
Modulation performance of blue InGaN light-emitting diodes with p-type electron-blocking layer and n-type hole-blocking layer
}

\author{
Gui-Chu Chen and Zhao-Xiong Zhao \\ Department of electronic information, Zhao Qing University, Zhaoqing, Guangdong, \\ China, 526061 \\ E-mail:gchenbox@163.com
}

\begin{abstract}
We have fabricated two blue light-emitting diodes (LEDs) samples with p-type electron-blocking Layer and n-type hole-blocking Layer for the modulation speed measurement. The experimental results show HBL can improve the modulation speed better for blue LED. The energy bands and recombination rates of both LEDs are investigated numerically within the APSYS software. The superior speed performance of LED with HBL can be attributed to its higher electron/hole recombination rate, which is verified by the simulation.
\end{abstract}

Keywords: LED; Modulation Speed; EBL; HBL.

\section{Introduction}

InGaN multiple quantum well (MQW) light-emitting diodes are promising for replacing conventional incandescent and fluorescent lamps due to their inherent higher energy efficiencies and other advantages. [1-4] However, blue LEDs suffer from a rapid efficiency droop with increasing injection current, $[5,6]$ which is detrimental to their high-brightness applications. The published simulations show that the electron leakage may play an important role in the efficiency droop. Therefore, the insertion of an p-AlGaN electron-blocking layer (EBL) between the last QW barrier and the p-GaN cap layer has been suggested, with the hope that this wide band gap material layer acts as an EBL to stop the electrons escaping from the active region. On the other hand, this convetional method also prohibits the holes from being injected into the active region due to the increasing potential height induced by EBL with the band bending effect. [7,8] Yen et al.[9] were aware of the disadvantages of using the traditional p-AlGaN EBL and replaced EBL with a n-AlGaN hole-blocking layer (HBL) near the n-GaN buffer layer, and they proved that blue LEDs with HBL exhibit a more excellent static optical performance, such as the light outpower.

In this paper, we fabricate two InGaN LED samples with p-AlGaN EBL and 
n-AlGaN HBL, respectively. Modulation performance of both are investigated experimentally, and LED with HBL shows more rapid modulation velocity. Furthormore the energy bands and recombination rates of both LEDs are investigated numerically with the APSYS (advanced physical model of semiconductor devices) simulation software. The simulation results reveal that the enhanced carrier recombination rate results in the superior dynamic performance of LED with HBL.

\section{Structure and Parameters}

The original blue InGaN/GaN MQW LED is grown by using MOCVD on a c-plane sapphire substrate, followed by a $4-\mu \mathrm{m}$ thick $n-G a N$ layer (n-doping $=2 \times 10^{18} \mathrm{~cm}^{-3}$ ). The active region consists of six 2.5-nm thick $\mathrm{In}_{0.15} \mathrm{Ga}_{0.85} \mathrm{~N}$ QWs sandwiched by seven $10-\mathrm{nm}$ thick GaN barriers. On top of the active region are a 20 -nm thick $\mathrm{p}-\mathrm{Al}_{0.15} \mathrm{Ga}_{0.85} \mathrm{~N}$ EBL (p-doping $=5 \times 10^{17} \mathrm{~cm}^{-3}$ ) and a $0.2-\mu \mathrm{m}$ thick $\mathrm{p}-\mathrm{GaN}$ cap layer ( $\mathrm{p}$-doping $=5 \times 10^{17} \mathrm{~cm}^{-3}$ ). The device geometry is designed into a rectangular shape of $300 \mu \mathrm{m} \times 300 \mu \mathrm{m}$. The LED with HBL has a similar layer structure except for the conventional p-AlGaN EBL. The p-AlGaN EBL is removed while the 20-nm thick $\mathrm{n}-\mathrm{Al}_{0.15} \mathrm{Ga}_{0.85} \mathrm{~N}$ (n-doping $=2 \times 10^{18} \mathrm{~cm}^{-3}$ ) is placed between the active region and the $\mathrm{n}-\mathrm{GaN}$ buffer layer. The schematic diagrams of the both structures are depicted in Fig. 1.

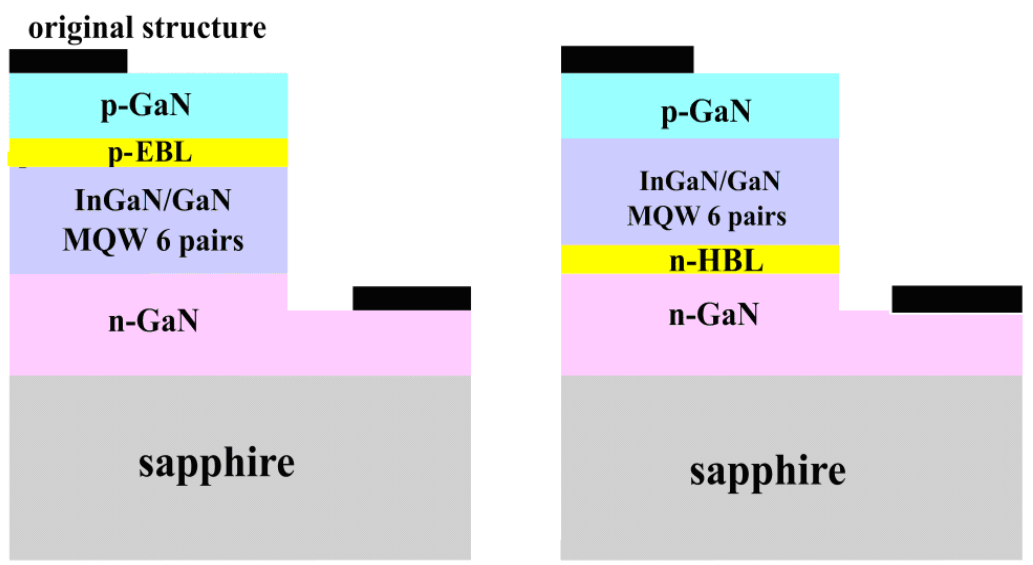

Fig.1 The schematic structures of two original LEDs with p-EBL and n-HBL

\section{Modulation Measurement}

During the modulation-speed measurement, we injected the RF signal into both LEDs and the modulated optical power was collected by lens and then fed into a 
low noise Si-based photoreceiver with a $1 \mathrm{G}-\mathrm{MHz}$ electrical bandwidth, which was connected with a RF spectrum analyzer. The influence of the used lens, photoreceiver, and bias tee, on the measured frequency response was de-embedded carefully. We denoted LED with EBL as Sample 1, and one with HBL as Sample 2. Fig. 3 shows the frequency responses of both samples under $50 \mathrm{~mA}$ bias current. We can clearly see that Sample 2 exhibits higher 3-dB electrical bandwidth than that of Sample 1 ( 24.5 MHz versus $\sim 21 \mathrm{MHz}$ ).

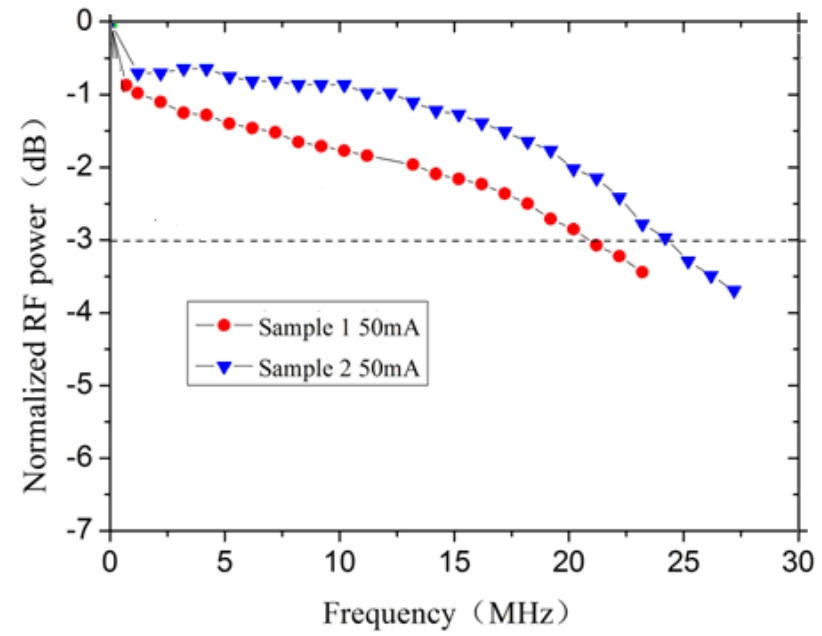

Fig. 2 Measured 3-dB frequency response of both samples under $50 \mathrm{~mA}$ bias current

Compared to the RC time constant of LED, the spontaneous recombination rate of carrier was proved to be a more dominant factor to affect the modulation speed of LED[9,10]. The energy bands and recombination rates of both LEDs with EBL and HBL are investigated numerically with the APSYS, which is capable of dealing with the physical properties of LEDs by solving Poisson's equation, the current continuity equation, the carrier transport equation, the quantum mechanical wave equation, and the photon rate equation.

\section{Simulation Results and Discussion}

Fig. 3 shows the band diagrams of both LEDs at $50 \mathrm{~mA}$. It can also be seen in Fig. 3(a) that the lattice mismatch between the last GaN QW barrier and AlGaN EBL can generate a strong strain, and then induce a strong piezoelectric polarization field, which pulls down the energy band at the last barrier and AlGaN EBL interface. As a result, the effective potential barrier height for electrons in the conduction band of EBL is reduced and the electron leakage can 
not be effectively suppressed. Moreover, because of the band bending effect, EBL also acts as an 812-meV height potential barrier for holes, which may hinder the holes from being injected into the active region. Fig. 3(b) shows the energy band diagrams of LED with HBL. The effective potential height is only $475 \mathrm{meV}$ for holes in the valance band near the last QW barrier, which implies that the holes inject more easily into the active region than those in the LED with HBL. Meanwhile the effective potential height (399 MeV) for electron injecting into the active region in LED with HBL is also lower than that 505 Mev) of LED with EBL. On the contrary, the effective potential barriers blocking electrons and holes in the active region in the EBL LED are higher than those of the HBL LED.
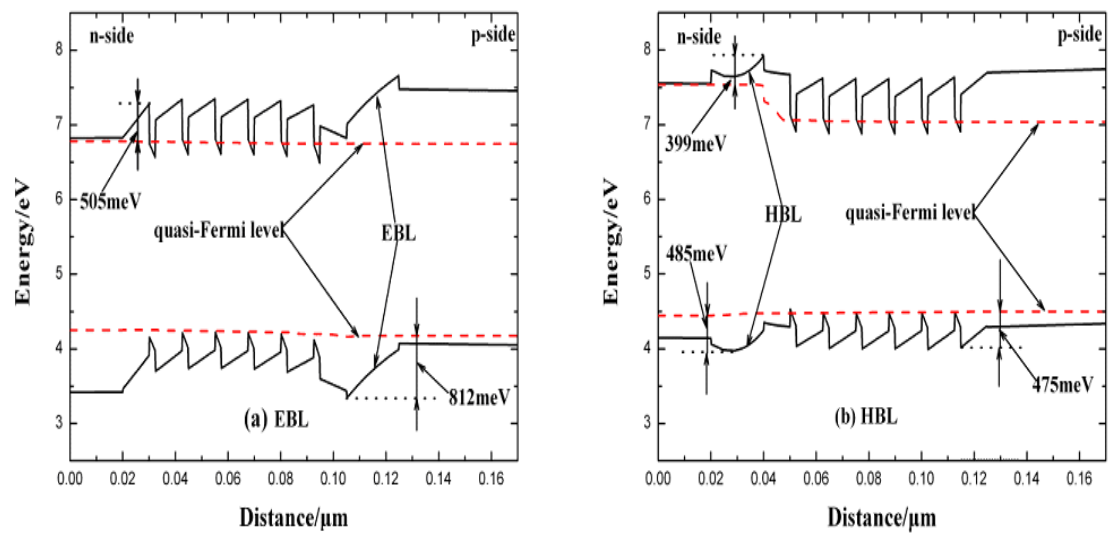

Fig. 3 The distance-dependent energy band diagrams of the LEDs with EBL and HBL

From the band informations mentioned above, we can conclude that the carriers inject more easily into the active region in LED with HBL, simultaneously the LED with EBL has less electrons and holes leakage. Fig. 4 shows the recombination rate simulated by the APSYS. We can see that the recombination rates are dominated by the QW close to the p-type layer resulting from nonuniform distributions of electrons and holes. The published simulations [11] show that the optical performance of blue LED is mainly dominated by the hole concentration rather than the electron concentration due to the low transfer velocity of the holes. As is shown in Fig. 4, owing to the enhancement of hole injection efficiency and the improvement of the efficiency of hole transport, the recombination rate of LED with $\mathrm{HBL}$ is higher than that of LED with EBL, which can explains our experimental results of both LEDs. 

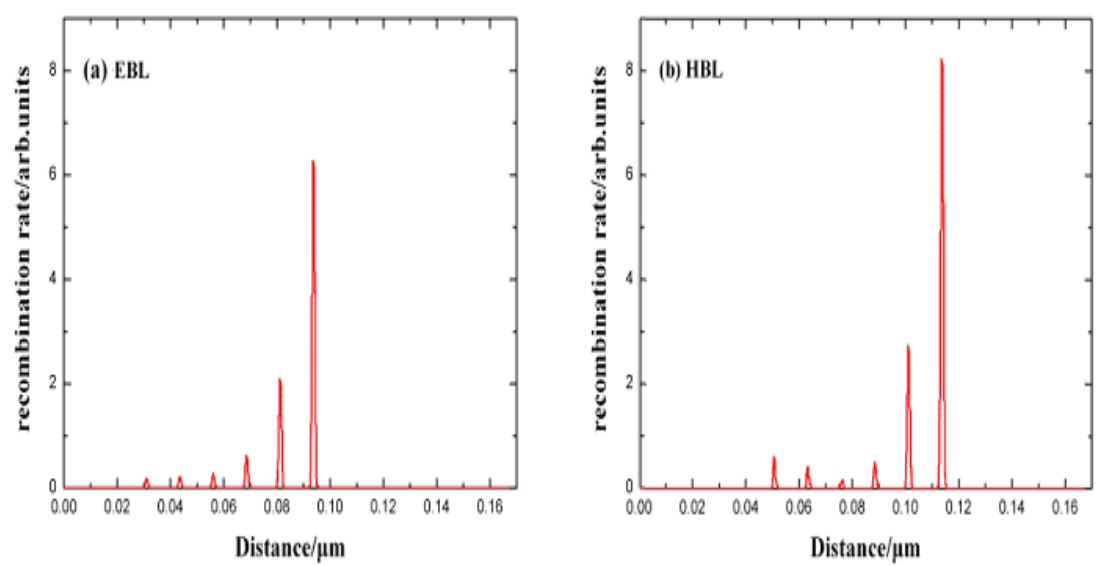

Fig. 4 The distance-dependent recombiantion rates of the LEDs with EBL and HBL

\section{Conclusion}

We have fabricated two LED samples with p-type EBL and n-type HBL, and their modulation velocity were measured by injecting the RF signal into the both LEDs.The reason of the superior modulation performance of LED with HBL to LED with EBL is the higher recombination rate in the former, which could be perfectly explained by the simulations induced by APSYS.

\section{References}

1. J. Shakya, K. Knabe, K. H. Kim, J. Li, J. Y. Lin, and H. X. Jiang, Polarization of III-nitride blue and ultraviolet light-emitting diodes, [J] Appl. Phys. Lett. 2005, 86:091107-091109,.

2. J. Li, J. Y. Lin, and H. X. Jiang, Growth of III-nitride photonic structures on large area silicon substrates, [J] Appl. Phys. Lett. 2006, 88:171909-171911.

3. Fan Y P, Cheng L W, Lin Y M, et al., Optimization of electrode shape for high power GaN-based light-emitting diodes, [J] Optoelectron. Lett., 2009, 5(5):0337-0340.

4. Kim K S, Kim J H, Jung S J, et al. Stable temperature characteristics of InGaN blue light emitting diodes using $\mathrm{AlGaN} / \mathrm{GaN} / \mathrm{InGaN}$ superlattices as electron blocking layer [J] Appl. Phys. Lett. 2010, 96(9):091104-1-3.

5. Y.-Y. Zhang and Y.-A. Yin, Performance enhancement of blue light-emitting diodes with a special designed $\mathrm{AlGaN} / \mathrm{GaN}$ supperlattice electron-blocking layer, [J] Appl. Phys. Lett., 2011, 99:221103-1-221103-3.

6. H. C. Hsu, Y. K. Su, S. J. Huang, C. Y. Tseng, C. Y. Cheng, and K. C. Chen, 
Enhanced performance of nitride-based blue LED with step-stage MQW structure, [J] IEEE Photon. Technol. Lett., 2011, 23(5):287-289.

7. C. Y. Jia et al., Performance improvement of GaN-based LEDs with step stage InGaN/GaN strain relief layers in GaN-based blue LEDs, [J] Opt. Exp., 2013, 21(7):8444-8449.

8. Z. Chen, W. Fenwick, and S. Lester, "Laterally contacted blue LED with superlattice current spreading layer,” U.S. Patent 8395165, Mar. 23, 2013.

9. Shi J-W, Huang H-Y, Sheu J-K, et al. The improvement in modulation speed of GaN-based green light-emitting diode (LED) by use of n-type barrier doping for plastic optical fiber (POF) communication [J] IEEE Photonics Technology Letters, 2006, 18(15): 1636-1638.

10. Shi J-W, Sheu J-K, Chen C-H, et al. High-speed GaN-based green light-emitting diodes with partially n-doped active layers and current-confined apertures [J]. IEEE Electron Device Letters, 2008, 29(2):158-160.

11. Kuo Y K, Chang J Y, Tsai M C, and Yen S H, Performance enhancement of blue light-emitting diodes by adjusting the p-type doped last barrier, Appl. Phys. Lett. 2009, 95:011116. 